\title{
Desempenho Social de Psicóticos em Função do Comportamento do Interlocutor ${ }^{1}$
}

\author{
Marina Bandeira \\ Sabrina Martins Barroso \\ Fernanda Valle Reis \\ Universidade Federal de São João Del Rei
}

\begin{abstract}
RESUMO
Foi investigado o desempenho social em função do comportamento do interlocutor, em uma amostra de 35 pacientes psicóticos comparativamente a 35 sujeitos não-clínicos. Dois observadores previamente treinados observaram os comportamentos dos sujeitos e dos interlocutores em 4 situações sociais de fazer e receber críticas, em desempenho de papéis, gravados em vídeo. Variou-se o tipo de situação e o gênero do interlocutor. Os resultados mostraram que o desempenho dos sujeitos estava positivamente relacionado aos comportamentos dos interlocutores de dar atenção, de dar feedbacks não-verbais e de olhar os sujeitos ao escutá-los e negativamente relacionado ao comportamento de olhar dos interlocutores nos momentos de silêncio. Os pacientes se mostraram mais sensíveis aos comportamentos dos interlocutores do que o grupo não clínico, pois seu desempenho se relacionou também positivamente com os feedbacks verbais do interlocutores. Estes resultados confirmam dados da literatura internacional da área e sugerem comportamentos específicos dos interlocutores como incentivo ao desempenho dos pacientes em programas de reabilitação.
\end{abstract}

Palavras-chave: competência social; psicóticos; reinserção social; desempenho de papéis.

\begin{abstract}
Social Skills of Psychotics and its Relations to Partners' Behaviors

This research investigated the social skills of psychotics in relation to its partners' behaviors in social interactions. Two trained judges observed a sample of 35 psychotics compared to 35 non-clinical subjects interacting with trained partners in 4 video-taped role-play situations, regarding giving and receiving criticism. Types of situations and gender of partners were varied. Results showed that subjects' behaviors were positively related to their partner's non-verbal feedbacks and looking behavior while listening to them and were negatively related to theirs partners' looking behavior during silence. Patients' group was more sensitive to partner's behaviors, their behaviors being also positively related to their partner's verbal feedbacks. These results replicated data from international literature in this area and suggest specific behaviors as incentive to the social skills of the patients in rehabilitation programsand suggest specific behaviors as incentive to the social skills of the patients in rehabilitation programs.
\end{abstract}

Keywords: social skills; partners' behavior; psychotics; desinstitucionalization; role-play.

\section{INTRODUÇÃO}

Em uma interação social, as reações da pessoa frente aos comportamentos do seu interlocutor constitui um aspecto importante a ser investigado, uma vez que esta informação pode contribuir para o desenvolvimento de programas de intervenção das habilidades sociais, podendo determinar o tipo de comportamento a ser privilegiado ou a ser evitado pelo interlocutor/treinador, visando a maximização da aprendizagem. Comportamentos do interlocutor que servem de feedbacks positivos para as pessoas, contribuindo para a emissão de comportamentos socialmente adequados, constituem componentes importantes da intervenção, juntamente com outras técnicas do treinamento de habilidades sociais, tais como ensaio comportamental, modelagem, modelação, instruções. Por outro lado, comportamentos do interlocutor/treinador que possuem funções aversivas de redução do comportamento da pessoa, com risco algumas vezes de se estender ao repertório da pessoa, devem ser evitados, visando a maximização da aprendizagem (Caballo, 1996; Del Prette \& Del Prette, 1999).

A reação das pessoas aos comportamentos do interlocutor pode variar dependendo das características da pessoa ou de suas condições psicopatológicas. No caso de pacientes psicóticos adultos, tal como o esquizofrênico, pode haver uma sensibilidade elevada às reações do interlocutor, em uma interação social. Considerando a dificuldade particular dos pacientes em enfrentar situações sociais (Cyr, Hodgins, Gaston 
\& Viens, 1990; Mercier, 1989), eles podem ser mais sensíveis a variações em alguns aspectos do comportamento do interlocutor, tais como os feedbacks verbais e não-verbais fornecidos, durante as interações sociais. Segundo Argyle (1984), os pacientes psiquiátricos, em particular o esquizofrênico, se mostram particularmente sensíveis a situações sociais negativas, por exemplo, quando são abordados temas conflituosos, conteúdos pessoais ou em situações em que são criticados.

Milbrook e Farrell (1986) constataram que a duração e a freqüência da fala e do olhar dos pacientes variavam em função das características dos comportamentos verbais e não-verbais do interlocutor, havendo, por exemplo, maior duração da fala dos pacientes quando o interlocutor olhava mais tempo para eles ao escutá-los, indicando atenção à sua fala ou quando o interlocutor apresentava mais comportamentos não-verbais de movimentar a cabeça, em sinal de aprovação à fala dos sujeitos.

A vulnerabilidade particular dos pacientes ao estresse interpessoal (Zubin \& Spring, 1977) e a sua ansiedade em interações sociais, podem torná-los mais dependentes da atenção e aprovação do interlocutor. As pausas durante a fala, os desvios de olhar, os silêncios e as hesitações, indicadoras de ansiedade, são comportamentos mais frequientes nestes pacientes, indicando assim sua vulnerabilidade e dificuldade nas interações sociais, que afetam seu desempenho social (Argyle \& Dean, 1965; Argyle \& Kendon, 1967; Nelson, Hayes, Felton \& Jarrett, 1985). Além disso, o déficit de assertividade apresentado por estes pacientes (Trower, 1980) pode torná-los mais dependentes dos feedbacks verbais e não-verbais do interlocutor que seriam demonstrativos de sua atenção e aprovação à fala dos pacientes (Bandeira \& Ireno, 2002). Por isto, é importante investigar as reações dos pacientes psiquiátricos em função de variações de comportamentos verbais e não verbais de interlocutores em interações sociais. Pesquisas avaliativas sobre este aspecto das reações dos pacientes podem contribuir para a identificação de comportamentos do interlocutor que afetam negativamente ou positivamente o desempenho dos pacientes, informação que pode favorecer o desenvolvimento de programas de treinamento das habilidades sociais mais adaptados para esta população-alvo, visando sua integração social e qualidade de vida.

A dificuldade de interagir com as pessoas tem sido considerada como um fator que afeta a integração de pacientes psiquiátricos em sua comunidade, interferindo no seu funcionamento social e comprometendo sua reinserção social. O déficit no funcionamento social tem sido constatado nos psicóticos em geral e no esquizofrênico, em particular, caracterizada pelo isolamento social e distúrbios no desempenho de papéis sociais, assim como dificuldade de estabelecer e manter relações sociais (Foy, Wallace \& Lieberman, 1983; Liberman, 1984). Dentro do contexto da desinstitucionalização psiquiátrica, o funcionamento social dos pacientes é considerado um elemento chave e tem sido colocado em destaque por pesquisadores de países nos quais a desinstitucionalização já completa cinco décadas de funcionamento (Bellack, Morrison, Mueser \& Wade, 1989; Test \& Stein, 1976).

Diversos fatores foram associados na literatura às dificuldades de reinserção social dos pacientes na comunidade e suas altas taxas de rehospitalizações (Bandeira \& Dorvil, 1996; Bandeira, 1993; Cyr, Toupin \& Lesage, 1992; Knapp, Chisholm, Astin, Lelliott \& Audini, 1997; Mercier, 1997; Rochefort, 1992). Dentre estes fatores, dois foram colocados em destaque por Stein, Diamond e Factor (1990) e por Test (1979), como principais determinantes das dificuldades encontradas na reinserção social dos pacientes: 1. O déficit de habilidades cotidianas e de habilidades de comunicação interpessoal dos pacientes e 2. A falta de um acompanhamento intensivo e diretivo. Segundo Test e Stein (1976), já está empiricamente demonstrado que o déficit das habilidades da vida cotidiana e de comunicação interpessoal é o fator que mais contribui para que o paciente se torne institucionalizado. Esta dificuldade em desempenhar as habilidades básicas do dia à dia e de se relacionar com o outro conduzem à frustrações constantes e ao estresse que, a longo termo, contribuem para as dificuldades de integração social e a ocorrência das rehospitalizações do paciente (Cyr e cols., 1992; Mercier, 1997; Test \& Stein, 1976).

A alta taxa de reinternações constitui um dos problemas mais citados no processo de desinstitucionalização psiquiátrica, conhecido como o Fenômeno da Porta Giratória (Bandeira \& Dorvil, 1996; Dorvil, 1987). Porém, o número e a gravidade das rehospitalizações dos pacientes diminui quando o seu nível de competência social é maior (Donahoe \& Drieseng, 1988; Hogarty, Anderson, Reiss, Kornblith, Greenwald, Ulrich \& Carter 1986; Liberman, Wallace, Blackwell, Eckman, Vaccaro \& Kuehnel, 1993; Shepperd, 1986).

Os programas de reabilitação psicossocial de pacientes psiquiátricos têm incluído sistematicamente atividades educativas visando capacitar os pacientes nestas habilidades básicas da vida cotidiana e nas habilidades de comunicação interpessoal. Destacamse, nesta área, o programa desenvolvido por Liberman e cols. (1993) e o programa de acompanhamento in- 
tensivo dos pacientes na comunidade, denominado PACT - Program for Assertive Community Treatment (Drake \& Burns, 1995; Test \& Stein, 1976), ambos enfatizando o ensino destas habilidades como um aspecto essencial dos programas (Bandeira, Gelinas \& Lesage, 1998).

Além do desenvolvimento dos programas de intervenção, a importância destas habilidades tem incentivado também a realização de diversas pesquisas avaliativas que visam investigar, de forma precisa e sistemática, o comportamento dos pacientes psiquiátricos, com o objetivo de identificar as dificuldades específicas verbais e não-verbais de comunicação interpessoal apresentadas por eles (Bellack, Morrison, Mueser \& Wade, 1989; Trower, 1980).

Estudos foram realizados também para desenvolver instrumentos de medida, estratégias ou métodos de avaliação válidos e fidedignos das habilidades sociais junto aos pacientes psiquiátricos. O estudo destas habilidades tem sido realizado principalmente através da observação sistemática do comportamento dos pacientes em interações sociais, através de desempenho de papéis, tendo em vista as limitações dos pacientes para outros tipos de avaliação, assim como a maior validade e fidedignidade da estratégia de observação do comportamento para esta população-alvo (Bellack, Morrison, Mueser, Wade \& Sayer, 1990).

As medidas de avaliação das habilidades sociais, através de desempenho de papéis apresentaram maior validade para esta população-alvo de pacientes psiquiátricos, em termos de validade preditiva, discriminante e concomitante, assim como fidedignidade inter e intra-avaliadores (Bellack, 1983; Bellack e cols., 1990; Eisler, Hersen, Miller \& Blanchard, 1975; Mueser e cols., 1990; Mueser, Bellack, Douglas \& Morrison, R.I., 1991; Merluzzi \& Biever, 1987). As situações sociais empregadas, nos desempenhos de papéis, são variadas, devido à especificidade situacional das habilidades sociais, que se refere a desempenhos e não a traço (Caballo, 1996; Del Prette \& Del Prette, 1999). Cyr e cols. (1990) destacaram a importância do estudo do comportamento dos pacientes em situações sociais conflituosas, devido às suas dificuldades particulares nestas situações. Tremblay (1992) destacou, em particular, as situações de fazer e receber crítica, que diferenciaram mais o grupo de pacientes de um grupo não-clínico. Estas situações serão, portanto, utilizadas no presente trabalho.

Os resultados das pesquisas, nesta área, apontaram diversos déficits dos pacientes nos componentes verbais e não-verbais da comunicação interpessoal. Por exemplo, foi observada a dificuldade de expressar insatisfação com o comportamentos das outras pessoas e de solicitar mudanças de comportamentos, assim como déficits nos componentes paralingúisticos e nãoverbais, tais como a duração da fala, o volume da voz, o comportamento de olhar, os gestos de apoio à fala, a expressividade, articulação, clareza e rítmo da fala, dentre outros aspectos (Bellack e cols., 1989; Bellack e cols., 1990; Donahoe \& Driesenga, 1988; Liberman, 1984; Mueser, Bellack, Douglas \& Morrison, 1991; Trower, 1980).

Pesquisas realizadas no Brasil confirmaram os dados obtidos na literatura internacional referentes a estas dificuldades dos pacientes psicóticos, constatando por exemplo, a presença de déficits nas habilidades verbais, não verbais e paralinguísticas dos pacientes, em interações sociais. Os pacientes apresentaram dificuldades de expressar insatisfação e de solicitar mudanças no comportamento do interlocutor e utilizaram conteúdos da fala incompletos e descontextualizados. Apresentaram ainda déficits nos aspectos não-verbais e paralinguísticos, tais como um volume de voz mais baixo, menor duração da fala, maior freqüência e duração de silêncios (Bandeira, Machado \& Pereira, 2002), freqüência menor de gestos de apoio à fala e afeto menos modulado e menos congruente com a situação (Bandeira, Cardoso, Fernandes, Resende \& Santos, 1998). Apresentaram peculiaridades na duração e frequiência do comportamento de olhar entre as fases de escuta e de elocução de interações sociais (Bandeira, Machado, Barroso, Gaspar \& Silva, 2002). Além disso, a clareza, articulação e ritmo da fala estavam igualmente deficitários (Bandeira e cols., 1998), assim como a impressão geral de competência social (Bandeira \& Tremblay, 1998) e de assertividade ou auto-afirmação na defesa de seus direitos (Bandeira e cols., 2002; Bandeira \& Ireno, 2002).

Não foram encontradas pesquisas, no contexto brasileiro, investigando o desempenho social de pacientes psiquiátricos em função das reações do interlocutor, em interações sociais. O objetivo da presente pesquisa é investigar estas relações, procurando identificar quais comportamentos dos interlocutores estão mais relacionados a um desempenho social mais competente dos pacientes e quais estão associados a um desempenho menos competente, comparativamente a pessoas sem história psiquiátrica, através da observação sistemática do comportamento, em desempenho de papéis.

\section{Participantes}

A amostra se constituiu de 70 sujeitos do sexo masculino, de nível socioeconômico baixo, da cidade de São João Del Rei e região, envolvendo dois grupos. 
O grupo clínico incluiu 35 pacientes psiquiátricos, com idade média de 39,40 anos (d.p.= 3,30), que moravam com suas famílias e que foram selecionados de arquivos de hospitais psiquiátricos públicos (Hospital Psiquiátrico da FHEMIG de Barbacena e Hospital Paulo Menicucci de Lavras). Estes pacientes haviam sido diagnosticados como psicóticos pelos psiquiatras dos hospitais (códigos 295 e 298). Foram excluídos os indivíduos que apresentavam retardo mental, distúrbios orgânicos ou que tiveram dificuldades de entender as instruções dadas durante as sessões experimentais. O Grupo não-clínico incluía 35 sujeitos sem história psiquiátrica, com idade média de 37,37 anos (d.p. = $11,19)$, que habitavam na mesma região geográfica dos pacientes, constituindo assim um grupo de referência dos pacientes na comunidade.

A equivalência dos dois grupos havia sido verificada previamente, com relação às variáveis idade, estado civil, renda familiar e per-capta, tipo de residência, nível de escolaridade e número de repetições escolares. Não havia diferenças significativas entre os grupos com relação a estas variáveis, o que possibilita uma maior validade interna da pesquisa e uma maior confiabilidade dos resultados (Bandeira \& Tremblay, 1998).

\section{Instrumento de Medida}

Os dados foram coletados através da observação sistemática do comportamento dos sujeitos em situações sociais de desempenho social, que já haviam sido previamente gravadas em vídeo. Os desempenhos de papéis haviam sido feitos com base na Escala de Avaliação de Competência Social, EACS (Bandeira, 2002), adaptada e validada para o Brasil, a partir de uma escala canadense de competência social (Tremblay \& Bandeira, 1989). A EACS consiste em um sistema de avaliação do comportamento através da observação sistemática, em quatro situações sociais cotidianas, nas quais são variados o gênero do interlocutor e as demandas das situações. A EACS inclui ainda a descrição dos cenários a serem utilizados em cada situação, os tipos de réplicas a serem usadas pelos interlocutores e as instruções a serem dadas aos participantes antes de iniciar os desempenhos de papéis, assim como os critérios de avaliação da competência social em uma escala tipo Likert de 6 pontos, crescentes quanto ao grau de competência social. (ver detalhes em Bandeira, 2002).

A observação era realizada por dois observadores previamente treinados e independentes, ou seja, cada um fazendo sua observação em momentos diferentes, na sala de observação para vídeos, visando assim minimizar a interferência entre eles.
Situações sociais: Foram utilizadas quatro situações de desempenho de papéis, representando interações sociais cotidianas. Em duas situações sociais, o sujeito devia fazer uma crítica ao interlocutor, expressando claramente sua insatisfação com o comportamento indesejável do interlocutor e solicitando uma mudança deste comportamento. No segundo tipo de situação, o sujeito devia reagir a uma crítica feita pelo interlocutor, reconhecendo sua responsabilidade, porém afirmando e justificando sua necessidade naquela situação e propondo uma solução para o problema, que satisfizesse a sua necessidade, porém sem desrespeitar a necessidade do outro. Para cada tipo de situação, variou-se o gênero do interlocutor.

Os sujeitos interagiam com interlocutores previamente treinados para este fim, os quais utilizavam réplicas múltiplas padronizadas porém flexíveis, que favoreciam uma maior naturalidade das interações sociais (Bandeira, 2002).

Categorias de comportamento observadas: Os comportamentos dos sujeitos e dos interlocutores foram observados nas diferentes fases das interações sociais: escuta, elocução e momentos de silêncio. Foram observados três comportamentos principais dos sujeitos, selecionados em função da sua importância no estudo das habilidades sociais e da possibilidade de comparação com outros estudos: 1) a duração da fala; 2) o comportamento de olhar (freqüência e duração), durante as fases de escuta e de elocução das interações sociais; 3) o comportamento de interromper a fala do interlocutor.

No que se refere ao interlocutor, foram selecionados os comportamentos que poderiam potencialmente ter um efeito sobre o comportamento dos sujeitos durante as interações sociais e que foram estudados na literatura da área: 1) frequiência de movimentos verticais de cabeça do interlocutor, na fase de escuta, indicativos de atenção à fala do sujeito; 2) freqüência de respostas verbais de feedback positivas do interlocutor, na fase de escuta, indicativas de aprovação ao discurso do sujeitos e encorajamento para que ele continuasse; 3) freqüência e duração do olhar do interlocutor em direção ao sujeito, nos momentos em que o está escutando, indicativos de atenção à fala do sujeito; 4) freqüência e duração do olhar do interlocutor enquanto está falando com o sujeito; 5) freqüência e duração do olhar do interlocutor durante os intervalos de silêncio que ocorrem entre as falas dos dois; 6) duração da fala do interlocutor; 7) freqüência do comportamento do interlocutor de interromper os sujeitos.

Foi utilizada igualmente uma medida do grau de competência social dos sujeitos, que já havia sido 
obtida anteriormente por dois observadores independentes, previamente treinados, que atribuíram pontos ao desempenho social dos sujeitos nas situações sociais estudadas. A competência social foi avaliada através de uma escala tipo Likert de 6 pontos, sendo o valor 1 considerado muito incompetente e o valor 6 muito competente.

\section{PROCEDIMENTO}

Treinamento dos observadores: Dois observadores foram treinados diariamente pelo pesquisador para observar, de forma precisa e fidedigna, os comportamentos dos sujeitos e dos interlocutores, em gravações de vídeo. Para o treinamento, foram utilizadas as gravações dos sujeitos que não participavam da pesquisa. Nas sessões diárias de treinamento, cada observador observava sozinho um sujeito por dia, por 1 hora aproximadamente. Em seguida, os observadores faziam uma sessão de discussão, calculando a porcentagem de acordos e desacordos obtidos. Os desacordos eram discutidos com o pesquisador, a cena era revista e buscava-se então identificar as causas dos desacordos, visando estabelecer critérios comuns de atribuição das cotas pelos dois observadores. $\mathrm{O}$ treinamento foi encerrado quando os dois observadores atingiram $85 \%$ de acordo em suas observações.

Coleta de dados: Após o treinamento, iniciou-se a coleta de dados, que teve a duração de 5 meses. Os observadores estavam "cegos" quanto ao grupo a que pertenciam os sujeitos, a fim de evitar o viés do observador na cotação dos comportamentos. Foi observado um sujeito por dia, para evitar que o cansaço dos observadores diminuísse sua capacidade de atenção e concentração. As sessões de observação dos vídeos eram diárias e tinham a duração média de 3 horas. Cada juiz observava os vídeos independentemente um do outro.

Durante as sessões de observação, cada observador avaliava cada uma das quatro situações sociais separadamente. Em cada situação social, observava-se um comportamento de cada vez, seguindo-se a ordem em que estavam listados na folha de registro de comportamentos. Observava-se o primeiro comportamento e anotava-se o seu valor na folha de registro. Em seguida, rebobinava-se a fita para observar e registrar o segundo comportamento, na mesma situação. Este procedimento era seguido para todos os outros comportamentos e visava facilitar a concentração dos observadores sobre um único comportamento, de forma a diminuir o risco de erros na observação, devidos a distração. Estes dados eram posteriormente utilizados para os cálculos de correlação entre os escores dos dois juízes, necessários à avaliação da fidedignidade das medidas ou acordo inter-observadores. Após a observação de um dado sujeito, os dois juízes calculavam a porcentagem de acordos obtidos entre eles, discutiam a respeito dos desacordos e chegavam à um consenso. O cálculo da porcentagem de acordos era feito diariamente, a fim de que este feedback contínuo contribuísse para manter um alto padrão de fidedignidade inter-observadores.

No caso de um desacordo, procurava-se chegar a um consenso que resultaria na medida final do comportamento em questão. Para as medidas de duração de um comportamento, que apresentavam uma dificuldade maior de avaliação, considerava-se como acordo entre os observadores, quando a diferença entre as suas medidas de duração, expressas em segundos e centésimos de segundo, se situava dentro da faixa de apenas $15 \%$ de diferença entre eles. Caso a diferença entre as duas medidas fosse superior a $15 \%$, considerava-se como desacordo. Neste caso, o consenso era obtido calculando-se a média das medidas dos dois observadores.

Para as medidas de frequiência, o consenso resultava de uma discussão entre os observadores a respeito dos critérios utilizados por cada um na observação do comportamento em desacordo. Para isto, eles voltavam à sala de observação e juntos refaziam a observação do comportamento em questão, discutindo sobre as causas dos desacordos até que chegassem a um consenso final. No caso em que não obtinham acordo entre eles, os juízes requeriam a presença do pesquisador e discutiam juntamente com ele qual critério seria o mais adequado para medir determinado comportamento, sempre levando em consideração as definições dos comportamentos estabelecidas durante a fase de treinamento. Em seguida, os dados do consenso eram anotados em uma folha de consenso específica para este fim.

Para se obter uma maior fidedignidade das medidas, foram adotados alguns procedimentos de coleta de dados. No caso das medidas de tempo, cada juiz cronometrava sempre três vezes o comportamento observado, adotando como escore de sua observação a mediana destas três medidas. Este procedimento visava diminuir o número de desacordos entre os juízes, uma vez que uma pequena diferença de centésimos de segundo nas medidas de duração era o suficiente para ultrapassar a margem dos $15 \%$ exigidos para se considerar as duas medidas como acordo.

No caso de algumas medidas de freqüência de comportamento que implicavam em maior dificuldade para os observadores (ex. freqüência do olhar nos intervalos em que não há fala) adotou-se o mesmo procedimento de coleta de dados descrito acima: cada 
juiz observava e contava três vezes um mesmo comportamento e considerava a mediana das três medidas como o escore final. Tal medida possibilitou uma maior segurança sobre a contagem da emissão dos comportamentos, evitando assim que um momento de desatenção por parte de um dos juízes gerasse um desacordo entre eles.

\section{RESULTADOS}

Os resultados obtidos serão apresentados separadamente para os dois grupos de sujeitos, tendo em vista os objetivos desta pesquisa. Os dados apresentaram um bom índice de fidedignidade das medidas de observação do comportamento. Os dados coletados através dos registros diários dos observadores obtiveram uma média de $96,60 \%$ de acordo entre eles. Os coeficientes de correlações de Pearson obtidos entre os escores dos observadores, para cada categoria de comportamento, foram todos significativos, variando entre $r=0,82$ e a $r=0,99(p<0,05)$.

\section{Grupo Clínico}

Os resultados referentes ao grupo clinico estão incluídos na Tabela 1, que apresenta as correlações de Pearson entre os comportamentos dos pacientes e as categorias selecionados do comportamento do interlocutor.

Comportamento de falar: Pode-se observar, pela Tabela 1, que foram obtidas correlações significativas entre o comportamento de falar dos pacientes e as principais categorias de comportamento do interlocutor que ocorriam simultaneamente à fala dos sujeitos. A duração da fala dos pacientes variou significativamente em relação às duas modalidades de feedbacks fornecidos pelos interlocutores. Os pacientes falavam por mais tempo, quando ocorria uma maior freqüência de feedbacks verbais dos interlocutores, indicativos de atenção à sua fala e também quando ocorria maior freqüência de feedback não-verbais, como movimentos verticais da cabeça, em sinal de compreensão da fala do pacientes.

Tabela 1: Correlações entre os comportamentos do interlocutor e dos sujeitos, apresentados pelo Grupo Clínico, no total das quatro situações Correlações entre os comportamentos do interlocutor e dos sujeitos

\begin{tabular}{|c|c|c|c|c|c|c|c|}
\hline Sujeito & $\begin{array}{l}\text { Duração da } \\
\text { Fala }\end{array}$ & $\begin{array}{l}\text { Freqüência do } \\
\text { olhar ao Ouvir }\end{array}$ & $\begin{array}{l}\text { Duração do } \\
\text { olhar ao } \\
\text { Ouvir }\end{array}$ & $\begin{array}{l}\text { Duração do } \\
\text { olhar ao } \\
\text { Falar }\end{array}$ & $\begin{array}{l}\text { Freqüência do } \\
\text { olhar ao Falar }\end{array}$ & $\begin{array}{l}\text { Freqüência de } \\
\text { Interrupções à fala } \\
\text { do Interlocutor }\end{array}$ & $\begin{array}{c}\text { Cota Global de } \\
\text { Competência } \\
\text { Social }\end{array}$ \\
\hline $\begin{array}{l}\text { Freqüência de } \\
\text { Feedback verbal } \\
\text { ao Ouvir }\end{array}$ & $0,50^{*}$ & $-0,10$ & 0,05 & $0,73^{\star \star}$ & $0,44^{*}$ & & $0,46^{*}$ \\
\hline $\begin{array}{l}\text { Freqüência de } \\
\text { movimentos de } \\
\text { Cabeça }\end{array}$ & $0,62^{* \star}$ & $-0,004$ & $-0,06$ & $0,73^{\star *}$ & $0,64^{* \star}$ & & $0,19^{*}$ \\
\hline $\begin{array}{c}\text { Freqüência de } \\
\text { olhares no Silêncio }\end{array}$ & $-0,37^{\star}$ & $-0,25$ & 0,08 & $-0,45^{\star}$ & $-0,46^{*}$ & & $-0,48^{*}$ \\
\hline $\begin{array}{l}\text { Duração do olhar } \\
\text { no Silêncio }\end{array}$ & $-0,40^{*}$ & $-0,32$ & 0,01 & $-0,46^{*}$ & $-0,48^{*}$ & & $-0,55^{\star *}$ \\
\hline $\begin{array}{l}\text { Freqüência de } \\
\text { olhares ao Ouvir }\end{array}$ & $0,56^{\star \star}$ & $0,36^{*}$ & 0,03 & 0,29 & $0,55^{\star \star}$ & & 0,20 \\
\hline $\begin{array}{c}\text { Duração do olhar } \\
\text { ao Ouvir }\end{array}$ & $0,96^{\star *}$ & 0,16 & $-0,16$ & $0,55^{\star \star}$ & $0,71^{\star *}$ & & 0,23 \\
\hline $\begin{array}{l}\text { Freqüência de } \\
\text { olhares ao Falar }\end{array}$ & 0,22 & $0,60^{\star \star}$ & $0,39^{\star}$ & 0,10 & 0,45 & & 0,10 \\
\hline $\begin{array}{l}\text { Duração do olhar } \\
\text { ao Falar }\end{array}$ & $-0,17$ & $0,36^{*}$ & 0,32 & $-0,20$ & $-0,23$ & & $-0,14$ \\
\hline $\begin{array}{l}\text { Duração da fala do } \\
\text { Interlocutor }\end{array}$ & 0,028 & $0,38^{\star}$ & $0,43^{\star *}$ & $-0,20$ & $-0,01$ & & $-0,12$ \\
\hline $\begin{array}{l}\text { Freqüência de } \\
\text { Interrupções }\end{array}$ & $0,84^{* \star}$ & & & & & $0,50^{*}$ & 0,31 \\
\hline
\end{tabular}

${ }^{* *} p \leq 0,001 ;{ }^{*} p \leq 0,05$ 
A duração da fala dos pacientes também variou significativamente em relação ao comportamento de olhar do interlocutor, enquanto os escutava. Os pacientes falavam por mais tempo, quando ocorria uma maior duração e frequiência do olhar dos interlocutores ao escutá-los, indicativos de mais atenção à fala dos pacientes.

Por outro lado, a duração da fala dos pacientes não se relacionou significativamente com categorias do comportamento do interlocutor que não ocorriam simultaneamente à fala dos sujeitos. Por exemplo, o fato do interlocutor olhar para os pacientes com maior ou menor freqüência e/ou duração, enquanto o próprio interlocutor falava, não se relacionou com o tempo de fala dos pacientes. A duração da fala dos pacientes também não apresentou relação significativa com o comportamento de falar do interlocutor, ou seja, os pacientes falavam mais tempo ou menos tempo, independentemente da duração da fala do interlocutor.

Uma correlação negativa significativa foi observada, entretanto, entre a duração da fala dos pacientes e os olhares dos interlocutores nos momentos de silêncio. Os pacientes falavam mais tempo, quando ocorria uma menor freqüência e duração dos olhares dos interlocutores, nos momentos de silêncio.

A duração da fala dos pacientes apresentou também uma relação positiva significativa com o comportamento de interromper do interlocutor, havendo maior frequiência de interrupções pelo interlocutor paralelamente a uma maior duração da fala dos pacientes.

Comportamento de olhar: Pode-se observar, pela Tabela 1, que foram obtidas correlações significativas entre o comportamento de olhar dos pacientes e as principais categorias do comportamento do interlocutor.

Com relação ao comportamento de olhar dos pacientes ao falar com o interlocutor, foi observado que a freqüência e duração destes olhares variaram significativamente em relação aos feedbacks verbais e nãoverbais simultâneos do interlocutor, esta correlação sendo mais elevada para a duração do que para a freqüência do olhar dos pacientes. Ou seja, os pacientes olhavam por mais tempo e mais frequentemente para o interlocutor paralelamente a uma maior freqüência de feedbacks verbais e não verbais dos interlocutores ao escutá-los. Este mesmo comportamento de olhar dos pacientes variou positivamente em relação a uma maior frequiência e duração dos olhares dos interlocutores ao ouvi-los. Por outro lado, uma correlação significativa inversa foi observada para os momentos de silêncio, indicando que uma maior duração e frequiência dos olhares dos pacientes ao falar com o in- terlocutor ocorria paralelamente a olhares menos freqüentes e menos demorados dos interlocutores nos momentos de silêncio.

No que se refere ao comportamento de olhar dos pacientes ao ouvir, a Tabela 1 mostra correlações positivas significativas, indicando que uma maior duração deste comportamento ocorria paralelamente ao comportamento simultâneo do interlocutor de falar mais tempo com eles e de olhar mais frequentemente para eles ao falar. A frequiência com que os pacientes olhavam para o interlocutor ao ouvi-lo também apresentou estas mesmas relações significativas e, além disso, variou positivamente em relação à freqüência dos olhares do interlocutor enquanto os ouvia.

Comportamento de Interromper: A Tabela 1 mostra uma correlação positiva significativa entre o comportamento dos pacientes de interromper a fala do outro e este mesmo comportamento emitido pelo interlocutor. Uma maior frequiência de interrupções feitas pelos pacientes ocorria paralelamente a uma maior frequiência de interrupções emitidas pelos interlocutores.

Grau de competência social: Foram obtidas correlações significativas relacionando o grau de adequação do desempenho dos pacientes, em termos de sua competência social, e algumas categorias do comportamento dos interlocutores. A Tabela 1 mostra que um desempenho mais competente dos pacientes se relacionou positivamente com os comportamentos do interlocutor de dar mais feedbacks verbais e não-verbais aos pacientes. Ao contrário, um desempenho mais competente dos pacientes se relacionou negativamente com o comportamento de olhar do interlocutor nos momentos de silêncio.

\section{Grupo Não-Clínico}

Os dados referentes ao grupo não-clínico encontram-se na Tabela 2, que inclui as correlações de Pearson entre os comportamentos dos sujeitos e seus interlocutores.

Comportamento de falar: Neste grupo de sujeitos, também se pode observar, pela Tabela 2, correlações significativas entre o comportamento de falar dos sujeitos e as principais categorias estudadas do comportamento do interlocutor. Porém, foi obtido um menor número de correlações do que para o grupo de pacientes. Por exemplo, a duração da fala dos sujeitos só variou significativamente em relação aos feedbacks não-verbais dos interlocutores, mas não teve relação com os feedbacks verbais dos interlocutores. 
Tabela 2: Correlações entre os comportamentos do interlocutor e dos sujeitos, apresentados pelo Grupo Não-Clínico, no total das quatro situações Correlações entre os comportamentos do interlocutor e dos sujeitos

\begin{tabular}{|c|c|c|c|c|c|c|c|}
\hline Sujeito & $\begin{array}{l}\text { Duração da } \\
\text { Fala }\end{array}$ & $\begin{array}{l}\text { Freqüência do } \\
\text { olhar ao Ouvir }\end{array}$ & $\begin{array}{c}\text { Duração do } \\
\text { olhar ao } \\
\text { Ouvir }\end{array}$ & $\begin{array}{l}\text { Duração do } \\
\text { olhar ao } \\
\text { Falar }\end{array}$ & $\begin{array}{l}\text { Freqüência do } \\
\text { olhar ao Falar }\end{array}$ & $\begin{array}{l}\text { Freqüência de } \\
\text { Interrupções à fala } \\
\text { do Interlocutor }\end{array}$ & $\begin{array}{c}\text { Cota Global de } \\
\text { Competência } \\
\text { Social }\end{array}$ \\
\hline $\begin{array}{c}\text { Freqüência de } \\
\text { Feedback verbal } \\
\text { ao Ouvir }\end{array}$ & 0,23 & $0,40^{*}$ & $0,39^{*}$ & 0,35 & 0,34 & & $0,50^{*}$ \\
\hline $\begin{array}{l}\text { Freqüência de } \\
\text { movimentos de } \\
\text { Cabeça }\end{array}$ & $0,82^{* *}$ & 0,28 & 0,22 & $0,61^{\star *}$ & $0,75^{\star \star}$ & & $0,46^{*}$ \\
\hline $\begin{array}{c}\text { Freqüência de } \\
\text { olhares no Silêncio }\end{array}$ & $-0,33$ & $-0,07$ & $-0,07$ & $-0,49^{*}$ & $-0,43^{*}$ & & $0,52^{*}$ \\
\hline $\begin{array}{l}\text { Duração do olhar } \\
\text { no Silêncio }\end{array}$ & $-0,45^{\star}$ & $-0,17$ & $-0,15$ & $-0,52^{*}$ & $-0,48^{*}$ & & $-0,67^{\star *}$ \\
\hline
\end{tabular}

cont.

\begin{tabular}{c|c|c|c|c|c|c|c}
\hline Sujeito & $\begin{array}{c}\text { Duração da } \\
\text { Fala }\end{array}$ & $\begin{array}{c}\text { Freqüência do } \\
\text { olhar ao Ouvir }\end{array}$ & $\begin{array}{c}\text { Duração do } \\
\text { olhar ao } \\
\text { Ouvir }\end{array}$ & $\begin{array}{c}\text { Duração do } \\
\text { olhar ao } \\
\text { Falar }\end{array}$ & $\begin{array}{c}\text { Freqüência do } \\
\text { olhar ao Falar }\end{array}$ & $\begin{array}{c}\text { Freqüência de } \\
\text { Interrupções à fala } \\
\text { do Interlocutor }\end{array}$ & $\begin{array}{c}\text { Cota Global de } \\
\text { Competência } \\
\text { Social }\end{array}$ \\
\hline $\begin{array}{c}\text { Freqüência de } \\
\text { olhares ao Ouvir }\end{array}$ & $0,36^{*}$ & $0,45^{*}$ & 0,26 & $-0,49^{*}$ & $-0,43^{*}$ & 0,32 \\
\hline $\begin{array}{c}\text { Duração do olhar } \\
\text { ao Ouvir }\end{array}$ & $0,96^{* *}$ & 0,24 & 0,12 & $0,66^{* *}$ & $0,74^{* *}$ & 0,29 \\
\hline $\begin{array}{c}\text { Freqüência de } \\
\text { olhares ao Falar }\end{array}$ & $-0,04$ & $0,42^{*}$ & $0,39^{*}$ & 0,05 & 0,22 & & 0,06 \\
\hline $\begin{array}{c}\text { Duração do olhar } \\
\text { ao Falar }\end{array}$ & $-0,01$ & $-0,04$ & 0,15 & $-0,03$ & $-0,11$ & & $-0,39^{*}$ \\
\hline $\begin{array}{c}\text { Duração da fala do } \\
\text { Interlocutor }\end{array}$ & $-0,03$ & $-0,04$ & 0,12 & $-0,04$ & $-0,12$ & & $-0,59^{* *}$ \\
\hline $\begin{array}{c}\text { Freqüência de } \\
\text { Interrupções }\end{array}$ & 0,31 & & & & & $0,49^{*}$ & 0,30 \\
\hline
\end{tabular}

${ }^{* *} p \leq 0,001 ;{ }^{*} p \leq 0,05$

A duração da fala dos sujeitos também variou significativamente em relação ao comportamento de olhar do interlocutor ao escutá-los. Os sujeitos falavam por mais tempo, paralelamente a uma maior duração e frequiência do olhar dos interlocutores ao escutá-los. Não houve correlação da fala dos sujeitos com a duração da fala dos interlocutores, nem com o comportamento de olhar dos interlocutores ao falar. Uma correlação negativa significativa foi observada, também para este grupo, entre a duração da fala dos sujeitos e os olhares dos interlocutores nos momentos de silêncio.

Contrariamente aos pacientes, não se observou neste grupo uma relação significativa entre a duração de sua fala e o comportamento de interromper do interlocutor.

Comportamento de olhar: Para este comportamento, o grupo não-clínico também apresentou correlações significativas em relação às principais cate- gorias do comportamento do interlocutor, embora menos do que os pacientes.

No que se refere ao comportamento de olhar dos sujeitos ao falar, foram observadas correlações positivas significativas da freqüência e duração destes olhares em relação aos feedbacks não-verbais simultâneos do interlocutor, mas não em relação aos feedbacks verbais, diferentemente dos comportamentos dos pacientes que se relacionaram a ambas as modalidades de feedback dos interlocutores.

Uma correlação significativa inversa também foi observada neste grupo, no que se refere aos momentos de silêncio, indicando uma maior duração e freqüência dos olhares dos sujeitos ao falar com o interlocutor, paralelamente a olhares menos freqüentes e menos demorados dos interlocutores nos momentos de silêncio.

No que se refere ao comportamento de olhar dos sujeitos ao ouvir, diferentemente dos pacientes, este grupo não apresentou correlações deste comportamento com a duração da fala dos interlocutores, mas 
sim com a duração do olhar simultâneo dos interlocutores ao falar com eles. A Tabela 2 indica ainda que uma maior duração do olhar dos sujeitos ao ouvir ocorria paralelamente a uma maior frequiência de feedbacks verbais simultâneos. Quanto à freqüência dos olhares dos sujeitos ao ouvir, foram observadas três correlações positivas significativas: em relação à freqüência dos feedbacks verbais do interlocutor, à frequiência dos seus olhares ao ouvir e à freqüência dos seus olhares ao falar com os sujeitos.

Comportamento de Interromper: Observou-se também para este grupo não-clínico que o comportamento dos sujeitos de interromper a fala do interlocutor apresentou uma correlação positiva significativa em relação a este mesmo comportamento emitido pelo interlocutor.

Grau de competência social: Neste grupo nãoclínico, houve quatro correlações significativas relacionando algumas categorias do comportamento dos interlocutores e o grau de competência social atribuído ao desempenho dos sujeitos. A Tabela 2 mostra que o um desempenho mais competente deste grupo se relacionou positivamente com os comportamentos do interlocutor de dar mais feedbacks verbais e nãoverbais aos sujeitos e se relacionou negativamente com a duração do olhar do interlocutor nos momentos de silêncio. Além disso, o desempenho mais competente deste grupo também se relacionou negativamente com a duração da fala do interlocutor e com a duração do olhar do interlocutor ao falar.

\section{DISCUSSÃO}

Os resultados da presente pesquisa mostraram que houve uma relação estreita entre os comportamentos dos sujeitos e dos interlocutores, no que se refere tanto ao comportamento de falar quanto de olhar dos sujeitos, assim como ao grau de competência social dos sujeitos.

Quanto ao comportamento de falar, foi observado que a duração da fala dos sujeitos, em ambos os grupos, era positivamente correlacionada com o comportamento não-verbal simultâneo do interlocutor, em particular com a duração do olhar dos interlocutores ao ouvi-los e com a frequiência dos feedbaks nãoverbais positivos fornecidos pelo interlocutor.

Apesar da limitação da estratégia correlacional para a interpretação dos resultados, algumas tendências podem ser inferidas, dentro dos limites desta análise. Os resultados descritos acima poderiam ser interpretados como um efeito de incentivo dos olhares e dos feedbacks não-verbais dos interlocutores ao escutar os sujeitos, uma vez que estes comportamentos são indicativos de atenção e compreensão do discurso do sujeitos, o que estimularia sua fala (Argyle \& Kendon, 1967). Estes resultados confirmam dados de Milbrook e Farrell (1986), pois estes autores encontraram também um tempo de fala maior dos sujeitos quando o interlocutor olhava mais tempo para eles e lhes fornecia mais feedbacks contingente à sua fala. Os resultados obtidos aqui colocam em destaque, portanto, a importância dos comportamentos não-verbais do interlocutor em uma interação social, em particular o olhar e os movimentos de cabeça, para estimular a fala dos sujeitos.

No que se refere ao grupo dos pacientes, especificamente, seu comportamento se mostrou ainda mais relacionado ao do interlocutor, pois foi obtido um maior número de correlações que não foram encontradas para o grupo não-clinico. Por exemplo, a duração da fala dos pacientes se relacionou, não só com a duração, mas também com a freqüência do olhar do interlocutor, nos momentos de silêncio. Observou-se, neste caso, que a duração da fala dos pacientes era menor, quando os interlocutores os olhavam mais tempo e mais frequentemente durante os momentos de silêncio.

Pode-se interpretar os resultados acima como indicativos de um efeito aversivo do olhar do interlocutor nos momentos de silêncio. Já foi observado, por exemplo, que pacientes esquizofrênicos apresentam uma vulnerabilidade particular e ansiedade nas situações sociais, se mostrando sensíveis à certos comportamentos dos interlocutores, tais como fazer críticas ou falar de temas emocionais ou pessoais, o que leva os pacientes a evitar mais o olhar dos interlocutores nestas situações negativas (Argyle \& Kendon, 1967; Cyr e cols., 1990; Zubin \& Spring, 1988). Além disso, segundo Argyle e Dean (1965), olhares prolongados demais, acima da faixa usualmente encontrada (entre 1 e 7 segundos), geram ansiedade nas pessoas e, em particular, nos pacientes psiquiátricos, tendo em vista sua vulnerabilidade particular. O comportamento de olhar dos interlocutores nos momentos de silêncio parece ter tido, portanto, um efeito aversivo, uma vez que não se relacionou a um aumento da fala dos sujeitos, ao contrário, estava correlacionado com uma menor duração da fala dos sujeitos. Deste modo, este comportamento do interlocutor não se mostrou útil, não sendo portanto recomendável em interações sociais com pacientes psiquiátricos.

O comportamento de falar dos pacientes se relacionou também com o comportamento verbal simultâneo do interlocutor, o que não ocorreu para o grupo não-clínico. Por exemplo, apenas para o grupo clínico observou-se que a duração da sua fala era maior quando os interlocutores davam mais feedback verbal ao ouvi-los. Estes resultados confirmam dados de Milbrook e Farrell (1986), pois em sua pesquisa os pacientes também falavam por mais tempo quando seus interlocutores utilizavam mais frequentemente o feedback verbal enquanto os escutavam. Estes resulta- 
dos poderiam ser interpretados como uma sensibilidade maior dos pacientes ao comportamento verbal dos interlocutores, devido ao fato deste comportamento ser indicativo de atenção, compreensão ou mesmo aprovação ao discurso dos pacientes. Do mesmo modo que os pacientes esquizofrênicos se mostram particularmente sensíveis a comportamentos verbais negativos dos interlocutores, tais como críticas, temas emocionais ou pessoais (Argyle \& Kendon, 1967), é possível que esta maior sensibilidade se estenda também a conteúdos verbais de aprovação do interlocutor. Além disso, tendo em vista que os pacientes psiquiátricos apresentam em geral um déficit de competência social e de comportamento assertivo (Bandeira \& Ireno, 2002; Bandeira e cols., 2002), este déficit pode torná-los mais sensíveis ou mais dependentes das reações apresentadas pelo interlocutor, em particular a freqüência do feedback positivo fornecido por ele, em uma interação social.

Um outro resultado observado apenas para o grupo clínico foi que os interlocutores interrompiam mais os pacientes quando eles falavam por mais tempo. Este resultado pode ser interpretado em função da tendência dos pacientes de apresentarem conteúdos verbais descontextualizados, incompletos e com uma freqüência menor de soluções para o problema discutido com o interlocutor, contrariamente ao grupo não-clínico (Bandeira, Barroso, Reis, Gaspar \& Silva, 2002).

Já foi observado, na literatura da área, que a duração da fala de uma pessoa é maior quando a duração da fala de seu interlocutor é menor, as duas variáveis sendo portanto negativamente relacionadas na população geral (Del Prette \& Del Prette, 1999). Por exemplo, Milbrook e Farrell (1986) obtiveram uma correlação negativa significativa entre estas duas variáveis, pois em sua pesquisa os sujeitos falavam mais tempo quando a duração da fala do interlocutor era menor e também quando a latência do interlocutor era menor. $\mathrm{Na}$ presente pesquisa, foi observada apenas uma tendência não-significativa dos dados nesta mesma direção, talvez devido ao número pequeno de sujeitos, tendo em vista que a análise de dados foi feita separadamente para cada grupo.

No que se refere ao comportamento de olhar dos sujeitos, os resultados mostraram que, em ambos os grupos, a frequiência e duração do olhar dos sujeitos enquanto estavam falando, se relacionaram positivamente com o comportamento não-verbal simultâneo dos interlocutores (olhar e movimentos de cabeça) ao escutá-los e também se relacionaram negativamente com o olhar do interlocutor nos momentos de silêncio. Os pacientes apresentaram uma relação mais estreita com o comportamento do interlocutor, pois para eles houve uma relação positiva também com o feedback verbal do interlocutor, o que não ocorreu com o grupo não clínico, que se mostrou mais independente. No que se refere ao olhar dos sujeitos ao ouvir o interlocutor, foi observado igualmente relações positivas com o comportamento simultâneo do interlocutor, referente à freqüência do seu olhar. No caso dos pacientes, também houve uma relação positiva com a duração do olhar do interlocutor e com a duração da fala do interlocutor. Estes resultados confirmam dados obtidos por Milbrook e Farrell (1986), segundo os quais a duração do olhar dos sujeitos era maior quando os interlocutores falavam por mais tempo, quando balançavam mais a cabeça em atenção à fala do sujeito e quando olhavam por mais tempo para eles ao escutá-los.

Os resultados obtidos na presente pesquisa sobre o comportamento de olhar confirmam dados obtidos previamente (Bandeira e cols., 2004) e corroboram a observação de outros autores (Argyle \& Dean, 1965; Argyle \& Kendon, 1967; Trower, 1980), demonstrando a necessidade de se estudar o comportamento de olhar separadamente nas fases de escuta, de elocução e de silêncio dos episódios de interação social, uma vez que este comportamento em cada fase apresenta diferentes variações em função de variáveis ambientais. Estes resultados estão em acordo com observações anteriores de que o comportamento de olhar, dependendo da fase, apresenta diferentes características de duração e freqüência e funções diferenciadas no processo de interação social e no processamento de informação, afetando a elaboração cognitiva necessária para construir respostas de solução de problemas interpessoais (Argyle \& Dean, 1965; Argyle \& Kendon, 1965; Caballo, 1996; Kendon, 1967).

No que se refere ao grau de competência social, os resultados obtidos aqui mostraram um desempenho mais competente dos sujeitos paralelamente a uma maior freqüência de feedbacks verbais e não-verbais do interlocutor. Estes resultados, em geral, confirmam dados de Milbrook e Farrell (1986), pois em sua pesquisa também foi encontrado um maior grau de competência no desempenho social dos sujeitos, quando os interlocutores apresentavam maior duração do olhar ao ouvi-los e uma latência mais curta. Este resultado pode ser interpretado como um efeito de incentivo dos feedbacks verbais e não verbais dos interlocutores, pois estes feedbacks consistem em sinais de aprovação e atenção aos comportamentos dos sujeitos, podendo estimular e fortalecer os comportamentos adequados, aumentando sua freqüência e duração, o que afeta diretamente a avaliação global de competência atribuída ao desempenho social dos sujeitos. Estes últimos resultados mostram que os comportamentos do interlocutor estão relacionados não apenas com comportamentos específicos dos sujeitos, mas também com o seu desempenho global, avaliado em termos do grau de competência social em uma interação. 
Pode-se concluir, portanto, que os resultados da presente pesquisa confirmam dados da literatura da área sobre a reação dos pacientes aos comportamentos do interlocutor, independentemente do contexto cultural. Os resultados indicaram a importância dos feedbacks verbais e não-verbais do interlocutor nas interações sociais com pacientes psiquiátricos, particularmente os feedbacks verbais, que apresentaram uma relação mais acentuada com os comportamentos dos pacientes e menos com o grupo não clínico. Esta pesquisa aponta para os aspectos do comportamento do interlocutor que parecem atuar como facilitador e, ao contrário, os aspectos que parecem funcionar como inibidores do comportamento dos pacientes nas interações sociais. Pesquisas futuras, envolvendo a manipulação experimental destes comportamentos específicos dos interlocutores, poderão testar, de forma mais concludente, as relações observadas no presente trabalho.

Os resultados foram analisados tomando-se os dados globais de cada grupo de sujeitos, sem diferenciar cada situação social específica, nem o gênero do interlocutor, uma vez que o objetivo da pesquisa era avaliar a relação entre os comportamentos dos sujeitos e de seus interlocutores, em interações sociais, em geral. Além disso, uma análise diferenciada envolveria um grande número de dados, o que seria incompatível com a limitação do tamanho deste artigo. Pesquisas futuras poderão examinar esta questão.

Os resultados obtidos na presente pesquisa poderão servir de base para sugerir atitudes que deveriam ser adotadas pelos terapeutas ou educadores em programas de reabilitação social de pacientes psiquiátricos, a fim de maximizar sua aprendizagem de comunicação interpessoal, tendo em vista a importância do desempenho social na reinserção dos pacientes na comunidade, no contexto da desinstitucionalização psiquiátrica.

\section{REFERÊNCIAS}

Argyle, M. (1984). Social skills and the analysis of situations and conversations. Em C. R. Hollin \& P. Trower (Orgs.), Handbook of social skills training: clinical applications and new directions (pp. 185-216). New York: Pergamon Press.

Argyle, M. \& Kendon, A. (1967). The experimental analyses of social performance. Advances in experimental social psychology, 3, 55-98.

Argyle, M. \& Dean, J. (1965). Eye contact, distance and affiliation. Sociometry, 28, 289-304.

Bandeira, M. (2002). Escala de avaliação da competência social de pacientes psiquiátricos através de desempenho de papéis EACS. Avaliação Psicológica, 2, 159-171.

Bandeira, M. (1993). Reinserção de doentes mentais na comunidade: fatores determinantes das rehospitalizações. Jornal Brasileiro de Psiquiatria, 42(9), 491-498.

Bandeira, M.; Machado, E.; Barroso, S.; Gaspar, T. \& Silva, M. (2003). Competência social de psicóticos: o comportamento de olhar nas fases de escuta e de elocução de interações sociais. Estudos de Psicologia, 8(3), 479-489.
Bandeira, M.; Cardoso, C. S.; Fernandes, M. L.; Resende, R. A. \& Santos, S. C. A. S. (1998). Competência social de psicóticos: validação social de habilidades específicas. Jornal Brasileiro de Psiquiatria, 47(5), 217-226.

Bandeira, M.; Gelinas, D. \& Lesage, A. (1998). Desinstitucionalização: o programa de acompanhamento intensivo na comunidade. Jornal Brasileiro de Psiquiatria, 47(12), 627-640.

Bandeira, M. \& Ireno, E. (2002). Reinserção social de psicóticos: avaliação global do grau de assertividade em situações de fazer e receber críticas. Psicologia: Reflexão e Crítica, 15(3), 665675.

Bandeira, M. \& Tremblay, L. (1998). Competência social de psicóticos: um estudo de validação social. Jornal Brasileiro de Psiquiatria, 47(4), 185-192.

Bandeira, M.; Machado, E. L. \& Pereira, E. A. A. (2002). Reinserção social de psicóticos: componentes verbais e não-verbais do comportamento assertivo, em situações e fazer e receber críticas. Psicologia: Reflexão e Crítica, 15(1), 89-104.

Bandeira, M. \& Dorvil, H. (1996). Les consultations à la salle d'urgence psychiatrique: comparaison entre des échantillons brésiliens et québécois. Le médecin du Québec, 31(8), 59-69.

Bandeira, M.; Barroso, S. M.; Reis, F. V.; Gaspar, T. R. \& Silva, M. M (2002). Competência social de psicóticos: o comportamento de alternância da fala em situações de fazer e receber críticas. Em Associação Nacional de Pesquisa e Pós-Gradução em Psicologia [Resumo], Anais do Simpósio de Pesquisa e Intercâmbio Científico da ANPEPP (p. 122). Águas de Lindóia: Anppep.

Bellack, A. S. (1983). Recurrent problems in the behavioral assessment of social skill. Behavior Research and Therapy, 21(1), 29-41.

Bellack, A. S.; Morrison, R. L.; Mueser, K. T. \& Wade, J. H. (1989). Social competence in schizoaffective disorder, bipolar disorder, and negative and non-negative schizophrenia. Schizophrenia Research, 2, 391-401.

Bellack, A. S.; Morrison, R. L.; Mueser, K. T.; Wade, J. H. \& Sayer, S. L. (1990). Role play for assessing the social competence of psychiatric patients. Journal of Consulting and Clinical Psychology, 2(3), 248-255.

Caballo, V. E. (1996). Manual de técnicas de terapia e modificação do comportamento. São Paulo: Santos Livraria.

Cyr, M.; Hodgins, S.; Gaston, L. E. \& Viens, L. (1990). La vie au sein d'appartements surveillés pour patients psychiatriques chroniques. Revue Canadienne de Santé Mentale Communautaire, 9(1), 23-37.

Cyr, M.; Toupin, J. \& Lesage, A. (1992). L'évaluation des problèmes psychosociaux chez des personnes ayant des problèmes psychiatriques graves. Relatório de pesquisa apresentado ao Conseil Québecois de la Recherche Sociale (RS1378, 88). Montreal, Canadá.

Del Prette, Z. A. P. \& Del Prette, A. (1999). Psicologia das habilidades sociais: terapia e educação. Rio de Janeiro: Vozes.

Del Prette, A. \& Del Prette, Z. A. P. (2003). Habilidades sociais: desenvolvimento e aprendizagem. Questões conceituais, avaliação e intervenção. Campinas: Alínea Editora.

Donahoe, C. P. \& Driesenga, S. A. (1988). A review of social skills training with chronic mental patients. Em M. Hersen; R. M. Eisler \& P. M. Miller (Orgs.), Progress in behavioral modification (pp. 131-164). Newbury Park: Sage.

Dorvil, H. (1987). Les caractéristiques du syndrome de la porte tournante à l'hôpital Louis-H. Lafontaine. Santé Mentale au Québec, XII(1), 70-89. 
Drake, R. E. \& Burns, B. J. (1995). Special section on assertive community treatment: an introduction. Psychiatric Services, 46(7), 667-668.

Eisler, R. M.; Hersen, M.; Miller, P. M. \& Blanchard, E. B. (1975). Situational determinants of assertive behaviors. Journal of Consulting and Clinical Psychology, 43(3), 330-340.

Foy, D. W.; Wallace, C. J. \& Liberman, R. P. (1983). Advances in social skills training for chronic mental patients. Advances in Clinical Behavioral Analysis, 11, 232-248.

Hogarty, G. E.; Anderson, C. M.; Reiss, D. J.; Kornblith, S. J.; Greenwald, D. P.; Ulrich, R. F. \& Carter, M. (1986). Family psychoeducation, social skills training, and maintenance chemotherapy in the aftercare treatment of schizophrenia. Archives of General Psychiatry, 48, 340-347.

Knapp, M.; Chisholm, D.; Astin, J.; Lelliott, P. \& Audini, B. (1997). The cost consequences of changing the hospitalcommunity balance: the mental health residential care study. Psychological Medicine, 27, 681-692.

Liberman, R. P. (1984). Social skills training with relapsing schizophrenics: an experimental analysis. Behavior Modification, 8(2), 155-173.

Liberman, R. P.; Wallace, C. J.; Blackwell, C. A.; Eckman, T. A.; Vaccaro, J. V. \& Kuehnel, T. G. (1993). Innovations in skills training for the seriously mentally ill: the UCLA social and independent living skills modules. Innovations and Research, 2, 43-60.

Mercier, C. (1989). Conditions de vie et lieu de résidence. Santé Mentale au Québec, XIV(2), 158-171.

Mercier, C. (1997). Les réalités de la réinsertion sociale. L'information Psychiatrique, 8, 813-821.

Merluzzi, T. V. \& Biever, J. (1987). Role-playing procedures for the behavioral assessment of social skill: a validity study. Behavioral Assessment, 9, 361-377.

Milbrook, J. M. \& Farrell, A. D. (1986). Behavioral components of social skills: a look at subject and confederate behaviors. Behavior Assessement, 8, 203-220.

Mueser, K. T; Bellack, A. S.; Morrison, R. L. \& Wade, J. H. (1990). Gender, social competence and symptomatology in schizophrenia: a longitudinal analysis. Journal of Abnormal Psychology, 99(2), 138-147.

\section{Nota:}

${ }^{1}$ Esta pesquisa foi financiada pela FAPEMIG e pelo CNPq.
Mueser, K. T.; Bellack, A. S.; Douglas, M. S. \& Morrison, R. L. (1991). Prevalence and stability of social skills deficits in schizophrenia. Schizophrenia Research, 5, 167-176.

Rochefort, D. A. (1992). More lessons of a different kind: canadian mental health policy in comparative perspective. Hospital Community Psychiatry, 43, 1083-1090.

Shepperd, G. (1986). Social skills training and schizophrenia. Em C. R. Hollin \& P. Trower (Orgs.), Handbook of social skills training: clinical applications and new directions (pp. 9-37). New York: Pergamon Press.

Stein, L. I.; Diamond, R. J. \& Factor, R. M. (1990). A system approach to the care of persons with schizophrenia. Em M. I. Herz; S. J. Keith \& J. P. Docherty (Org.), Handbook of Schizophrenia, vol. 4, Psychosocial Treatment of Schizophrenia ( $p p$. 213-246). Amsterdam: Elsevier Sciences Publishers.

Test, M. A. \& Stein, L. I. (1976). Practical guidelines for the community treatment of markedly impaired patients. Community Mental Health Journal, 12(1): 72-82.

Test, M. A. (1979). Continuity of care in community treatment. Em Stein, L. I. (Org.), Community support systems for the longterm patient. New Directions for Mental Health Services, 2, 5766.

Tremblay, L. (1992). Évaluation de la competence sociale de personnes souffrant de troubles mentaux graves: études de validation sociale. Relatório de pesquisa apresentado ao Conseil Québecois de la Recherche Sociale (RS-1725-089). Montreal, Canadá.

Tremblay, L. \& Bandeira, M. (1989). Evaluation ecologique des habiletes sociales. Institut Philippe Pinel de Montréal. Manuscrito não publicado.

Trower, P. (1980). Situational analysis of the components and processes of behavior of socially skilled and unskilled patients. Journal of Consulting and Clinical Psychology, 48, 327-339.

Zubin, J. \& Spring, B. (1977). Vulnerability: a new view of schizophrenia. Journal of Abnormal Psychology86, 103-126.

Enviado: 28/07/2004 Revisado: 10/11/2004 Aceito: 13/12/2004

\section{Sobre as autoras:}

Marina Bandeira: Professora Adjunta da Universidade Federal de São João Del Rei (UFSJ). Pesquisadora do CNPq. Doutora pela Université de Montréal, Canadá.

Departamento de Psicologia - Laboratório de Pesquisa em Saúde Mental - LAPSAM - site: www.ufsj.edu.br/saudemental.

Endereço para correspondência: Praça Dom Helvécio, 74 - 36301-160 São João Del Rei - MG - Endereço Eletrônico: bandeira@ufsj.edu.br.

Sabrina Martins Barroso: Psicóloga formada pela UFSJ.

Fernanda Valle Reis: Aluna do curso de graduação em Psicologia da UFSJ. 